\title{
Leukotriene receptor antagonist therapy and Churg-Strauss syndrome: culprit or innocent bystander?
}

\author{
Richard Beasley, ${ }^{1}$ Susan Bibby, ${ }^{1}$ Mark Weatherall ${ }^{2}$
}

Randomised controlled trials can provide strong evidence of the efficacy (or lack) of new drug treatments as well as the occurrence of common side effects. ${ }^{1}$ However, clinical safety issues can arise after new medications receive regulatory approval, particularly in relation to rare serious adverse events. ${ }^{2}$ Investigation of rare adverse events is often fraught with difficulty, leading to uncertainty, particularly when there is conflicting evidence from research utilising different methodologies. One recent example is the observed association between leukotriene receptor antagonist (LTRA) therapy, used in the treatment of asthma, and ChurgStrauss syndrome (CSS), a vasculitis of uncertain aetiology (also known as allergic granulomatous angiitis). CSS is certainly both rare and serious, with a background incidence of 3 per million per year in the general population, and a 1 year mortality rate of $7 \%$. $^{3-5}$

Soon after the introduction of LTRAs (zafirlukast, pranlukast and montelukast), numerous case reports and case series were published of patients who developed CSS after starting this therapy. ${ }^{6-13}$ The temporal relationship between the introduction of LTRA therapy and the development of CSS suggested a possible causal relationship. An underlying mechanism was proposed whereby LTRA therapy may lead to an imbalance in leukotriene receptor stimulation, resulting in unopposed activity of $\mathrm{LTB}_{4}$, a potent chemoattractant for eosinophils as well as neutrophils, which could potentially lead to eosinophilic tissue infiltration and the initiation of systemic vasculitis. $^{14} 15$

A feature of a number of the case reports was that the introduction of the

\footnotetext{
${ }^{1}$ Medical Research Institute of New Zealand, Wellington, New Zealand; ${ }^{2}$ University of Otago Wellington, Wellington, New Zealand

Correspondence to: Professor Richard Beasley, Medical Research Institute of New Zealand, PO Box 10055, Wellington 6143, New Zealand;

Richard.Beasley@mrinz.ac.nz
}

LTRA allowed significant oral steroid reduction, suggesting that this therapy may have unmasked previously existing CSS that had been suppressed by the steroids prescribed for asthma. Another observation was that some cases had severe or unstable asthma at the time LTRA therapy was introduced, which arguably may have represented the earliest phase of CSS, characterised by asthma and rhinosinusitis. It has been proposed that in these cases, LTRA therapy may have been prescribed in response to the initial phase of CSS, which then progressed to eosinophilia with pulmonary infiltration and subsequently systemic vasculitis during LTRA therapy. ${ }^{16}$ It has been difficult to assess the validity of these hypotheses because of the paucity of data that has been available, limited primarily to either case reports or case series in which the method of case selection was not specified, giving potential for selection and publication bias.

These hypotheses were not supported by the observation that there were very few published reports of CSS following the introduction of other asthma treatments, including inhaled corticosteroids (ICS), theophylline, cromolyns and long acting $\beta$ agonists (LABAs), all of which have shown some steroid sparing effects. ${ }^{13}$ Similarly, of 63 cases of CSS reported to the UK Committee on Safety of Medicines (CSM) through the Yellow Card Scheme since 1963, 59 were documented in 1998 and 1999; of these, $90 \%$ were associated with drugs used to treat asthma, mainly LTRAs. ${ }^{17}$ Likewise, in the US FDA Adverse Event Report System database, reporting of CSS was strongly associated with LTRA use and not other commonly used asthma therapies, including ICS and LABA therapy. ${ }^{18}$ ICS are more likely to have oral steroid sparing effects, ${ }^{19}{ }^{20}$ and LABAs greater ICS sparing effects ${ }^{21} 22$ than LTRA therapy. ${ }^{23}$ Thus if CSS does indeed appear in response to steroid withdrawal, these commonly prescribed therapies are more likely than LTRAs to result in unmasking of CSS. Furthermore, the recommended use of ICS and LABA therapy in severe asthma ${ }^{24}$ might be expected to result in their greater coincident use in patients with pre-existing CSS manifest by severe unstable asthma.

To balance this interpretation there is recent evidence that the predominant association of CSS with LTRA and not with other commonly prescribed therapies may be due in part to selection bias. In a recent US based case control study, only 6/47 (13\%) patients with CSS were exposed to LTRA therapy, ${ }^{25}$ markedly lower than the proportion of CSS cases on LTRA reported to the FDA or CSM, or published in the medical literature. This study reported a strong association between LTRA use and CSS, but in multivariate analyses controlling for asthma drug use, no significant association was observed. It was acknowledged that because of the low power of the study, it was not possible to rule out an up to fourfold increased risk of CSS with LTRA therapy. There are also a number of recent case series reporting the occurrence of CSS unrelated to LTRA therapy, including cases in which ICS therapy allowed oral corticosteroid withdrawal. $^{26}{ }^{27}$ However, others have reported an increase in the incidence of CSS driven primarily by cases using LTRA therapy. ${ }^{28}$

As a result, there is conflicting evidence on whether the association between LTRA and CSS is primarily a result of confounding by indication, or represents a genuine causal association. Two papers, one published in this issue of Thorax, ${ }^{29}$ and the other published recently, ${ }^{30}$ utilising different methodologies, have addressed this issue and come to different conclusions (see page 883). Nathani et al present a systematic review of cases and cases series that report CSS in association with LTRA use. ${ }^{29}$ There were 62 patients from 40 publications which were identified from Medline included in the review. The main finding was that the majority of patients exhibited a clear temporal relationship between initiation of LTRA therapy and development of CSS, with no evidence of pre-existing disease or steroid withdrawal. Indeed, seven (11\%) cases were entirely steroid naïve, not on ICS or oral corticosteroids when LTRA therapy was initiated. Cases were identified in which there was a relapse of CSS following reintroduction of LTRA therapy, and other cases in which there was remission of signs and symptoms of CSS on withdrawal of LTRA therapy, which could not be attributed to systemic 
steroid or immunosuppressive therapy. The authors concluded that the available evidence suggests an association between LTRA and CSS that may be causal.

In the other publication, Hauser et al used an extension of case control methodology to investigate exposure to the LTRA montelukast (and other asthma medications) and the development of CSS. ${ }^{30}$ The case control study identified 78 patients from two databases, one a French cohort who were participating in a clinical trial of therapy for CSS, and the other a German cohort which was part of a prospective registry for a tertiary referral centre for vasculitis management. Both databases were accumulated over a 10 year period and the study focused on montelukast as it was the only LTRA approved in France and Germany. The main findings were that the use of montelukast in the 3 months before the development of CSS was more likely than use in the period more than 3 months before its development, and that positive estimates were also obtained for other long term asthma control medications with effects that were independent of montelukast use. The authors concluded that because the association with CSS onset was not specific to montelukast but also occurred with other asthma medications, it was more likely to be caused by a general escalation of treatment in response to worsening asthma. They also noted that there was increasing use of montelukast during the study period, adding weight to a confounding rather than causative role of montelukast in CSS development.

Both papers highlight the fact that CSS is rare, and that as a result, statistical testing is likely to lack power to detect important associations (highlighted by the wide confidence intervals in the case control study). Both studies are also likely to be influenced by selection bias, which may limit the generalisability of the findings. The evidence of causation provided by case reports and case series is usually prescribed lesser weight than that of case control studies. However, the systematic review by Nathani and colleagues $^{29}$ does provide important information that CSS may occur in association with LTRA therapy in the absence of preexisting disease, may occur on rechallenge with LTRA therapy and may remit on withdrawal of LTRA therapy without the requirement to start or increase the dose of systemic steroid or immunosuppressive therapy.

The evidence against causation provided by the case control study of Hauser and colleagues ${ }^{30}$ is open to debate.
The authors did in fact identify a strong association between CSS and LTRA therapy and were unable to convincingly demonstrate that it was confounding by indication that led to this association. In the multivariate analyses, in which all four asthma medication classes were included as covariates, the risk of developing CSS within 3 months was 6.7 (95\% confidence interval (CI) 1.3 to 34.1) for montelukast, 4.2 (95\% CI 1.2 to 14.6$)$ for oral steroids, 2.9 (95\% CI 0.6 to 13.3$)$ for LABAs and 1.0 (95\% CI 0.2 to 4.8) for ICS. Thus there was a significant risk associated with LTRA, but not with ICS and LABA therapy, although it must be noted that all the confidence intervals were wide, resulting in considerable uncertainty in interpretation. The increase in risk with oral steroids is likely to relate to their use in severe exacerbations of asthma, resulting from the progression of CSS. Notably, Hauser and colleagues ${ }^{30}$ have also provided substantive evidence (consistent with the previous case control study ${ }^{25}$ ) that there is likely to have been major selection bias in the reporting of the association between asthma therapy and CSS, in which the vast majority of reported cases relate to LTRA therapy, whereas such cases represented only a quarter of their database.

The study of rare serious adverse events associated with medication use is inherently difficult and the investigation of the association between LTRA therapy and CSS is no exception. However, despite the apparent conflicting nature of the data, it can be brought together in a consistent manner to conclude that there are a number of different clinical circumstances in which CSS may occur in association with LTRA therapy. These include its use for worsening asthma which may or may not be coincidentally a symptom of an early phase of a progressive pre-existing CSS; its use resulting in clinical improvement leading to a reduction in steroid use potentially allowing unmasking of underlying CSS; and its use in patients with no evidence of preexisting CSS or change in steroid use. In addition, its use may also lead to a recurrence of CSS and its withdrawal may be associated with a remission independent of systemic steroid or immunosuppressive therapy. There is now sufficient evidence to suggest that all of these distinct clinical scenarios may occur, with the priorities now to determine the proportion of cases in which the association is causative, and the pathophysiological processes by which this occurs. ${ }^{31}$ Doctors need to be mindful of the risk of CSS associated with LTRA therapy, particularly when prescribed to patients with moderate to severe asthma or in the setting of steroid withdrawal, and be aware of the clinical manifestations of CSS and the requirement to withdraw LTRA therapy should CSS develop.

Competing interests: RB has received fees for consulting and speaking and reimbursement for attending symposia from Astra Zeneca, GlaxoSmithKline and Novartis. SB and MW have no conflict of interest to declare.

Thorax 2008;63:847-849. doi:10.1136/thx.2007.093971

\section{REFERENCES}

1. Grimes DA, Schulz KF. An overview of clinical research: the lay of the land. Lancet 2002;359:57-61.

2. Maxwell SRJ, Webb DJ. COX-2 selective inhibitorsimportant lessons learned. Lancet 2005;365:449-51.

3. Churg A. Recent advances in the diagnosis of ChurgStrauss syndrome. Mod Pathol 2001;14:1284-93.

4. Noth I, Strek ME, Leff AR. Churg-Strauss syndrome. Lancet 2003;361:587-94.

5. Bourgarit A, Le Toumelin P, Pagnoux C, et al. Deaths occurring during the first year after treatment onset for polyarteritis nodosa, microscopic polyangiitis, and Churg-Strauss syndrome. Medicine 2005;84:323-30.

6. Knoell DL, Lucas J, Allen JN. Churg-Strauss syndrome associated with zafirlukast. Chest 1998;114:332-4.

7. Wechsler ME, Garpestad E, Flier SR, et al. Pulmonary infiltrates, eosinophilia, and cardiomyopathy following corticosteroid withdrawal in patients with asthma receiving zafirlukast. JAMA 1998;279:455-7.

8. Katz RS, Papernik M. Zafirlukast and Churg-Strauss syndrome. JAMA 1998;279:1949.

9. Kinoshita M, Shiraishi T, Koga T, et al. ChurgStrauss syndrome after corticosteroid withdrawal in an asthmatic patient treated with pranlukast. J Allergy Clin Immunol 1999;103:534-5.

10. Green RL, Vayonis AG. Churg-Strauss syndrome after zafirlukast in two patients not receiving systemic steroid treatment. Lancet 1999;353:725-6.

11. Franco J, Artés MJ. Pulmonary eosinophilia associated with montelukast. Thorax 1999;54:558-60.

12. Lanham JG, Elkon KB, Pusey CD, et al. Systemic vasculitis with asthma and eosinophilia: A clinical approach to the Churg-Strauss syndrome. Medicine (Baltimore) 1984;63:65-81.

13. Stirling RG, Chung KF. Leukotriene antagonists and Churg-Strauss syndrome: the smoking gun. Thorax 1999;54:865-6.

14. Guilpain P, Viallard J-F, Lagarde P, et al. ChurgStrauss syndrome in two patiens receiving montelukast. Rheumatology 2002;41:535-9.

15. Crooks SW, Stockley RA. Leukotriene B4. Int J Biochem Cell Biol 1998;30:173-8.

16. Wechsler ME, Pauwels R, Drazen JM. Leukotriene modifiers and Churg-Strauss syndrome: adverse effect or response to corticosteroid withdrawal? Drug Saf 1999;21:241-51.

17. Committee on Safety of Medicines/Medicines Control Agency. Leukotriene receptor antagonists: update on adverse reaction profiles. Curr Probl Pharmacovigilance 1999;25:14.

18. DuMouchel W, Smith ET, Beasley R, et al. Association of asthma therapy and Churg-Strauss syndrome: an analysis of postmarketing surveillance data. Clin Ther 2004;26:1092-104.

19. Nelson HS, Busse WW, de Boisblanc BP, et al. Fluticasone propionate powder: oral corticosteroid sparing effect and improved lung function and quality of life in patients with severe chronic asthma. J Allergy Clin Immunol 1999;103:267-75.

20. Nelson H, Berstein L, Fink J, et al. Oral glucocorticosteroid-sparing effect of budesonide administered by turbuhaler. Chest 1998:113:1264-71.

21. Busse W, Koenig SM, Oppenheimer J, et al. Steroidsparing effects of fluticasone propionate $100 \mu \mathrm{g}$ and salmeterol $50 \mu \mathrm{g}$ administered twice daily in a single product in patients previously controlled with fluticasone propionate $250 \mu \mathrm{g}$ administered twice daily. J Allergy Clin Immunol 2003;111:57-65. 
22. Lalloo UG, Malolepszy J, Kozma D, et al. Budesonide and formoterol in a single inhaler improves asthma control compared with increasing the dose of corticosteroid in adults with mild-to-moderate asthma. Chest 2003;123:1480-7.

23. Löfdahl C-G, Reiss TF, Leff JA, et al. Randomised, placebo controlled trial of effect of a leukotriene receptor antagonist, montelukast, on tapering inhaled corticosteroids in asthmatic patients. BMJ 1999;319:87-90.

24. British Thoracic Society, Scottish

Intercollegiate Guidelines Network. British
Guideline on the Management of Asthma. Thorax 2008;63(Suppl IV):iv1-121.

25. Harrald LR, Patterson MK, Andrade SE, et al Asthma drug use and the development of ChurgStrauss syndrome (CSS). Pharmacoepidemiol Drug Saf 2007;16:620-6.

26. Bili A, Condemi JJ, Bottone SM, et al. Seven cases of complete and incomplete forms of Churg-Strauss syndrome not related to leukotriene receptor antagonists. J Allergy Clin Immunol 1999;104:1060-5.

27. Le Gall C, Pham S, Vignes S, et al. Inhaled corticosteroids and Churg-Strauss syndrome: a report of five cases. Eur Respir J 2000;15:978-81.
28. Solans R, Bosch JA, Selva A, et al. Montelukast and Churg-Strauss syndrome. Thorax 2002:57:183-5.

29. Nathani N, Little MA, Kunst $\mathrm{H}$, et al. Churg-Strauss syndrome and leukotriene antagonist use: a respiratory perspective. Thorax 2008;63:883-8.

30. Hauser T, Mahr A, Metzler C, et al. The leukotriene receptor antagonist montelukast and the risk of Churg-Strauss syndrome: a case crossover study. Thorax 2008:63:677-82.

31. Weller PF, Plaut M, Taggart V, et al. The relationship of asthma therapy and Churg-Strauss syndrome: $\mathrm{NIH}$ workshop summary report. J Allergy Clin Imunol 2001;108:175-83.

\section{A breath of fresh air for acute oxygen treatment}

\section{Peter Calverley}

Oxygen therapy given to acutely ill people is one of the commonest interventions used in modern medicine and has become part of the folklore of our times as a sick patient wearing an oxygen mask is pushed through the emergency department, both in real life and on television. Although the principles of oxygen treatment have been established by painstaking quantitative research over the past 60 years, in practice most people learn to use oxygen by following customary practice in their institution rather than considering rationally how it is best employed. A feeling that some oxygen is good, therefore more must be better, can be a dangerous precept to follow, whereas an unnecessary paranoia about inducing carbon dioxide retention can deny some people potentially life saving treatment. These uncertainties make the arrival of the new British Thoracic Society Guideline for Emergency Oxygen Use in Adults $^{1}$ particularly welcome. This rather daunting document, which has been extensively endorsed by key professional societies and practitioner groups, provides a comprehensive and at times exhaustive review of the theory and practice of acute oxygen treatment. Its scope ranges from a detailed consideration of how oxygen therapy influences respiratory physiology and tissue oxygen delivery through to which mask should be chosen and how oxygen services should be developed. It is a major resource which answers almost any question about acute oxygen therapy but is best consulted selectively,

Correspondence to: Professor Peter Calverley, Department of Medicine, Clinical Sciences Centre, University Hospital Aintree, Longmoor Lane, Liverpool L9 7AL, UK; pmacal@liverpool.ac.uk depending on the interest and needs of the user. The helpful summary of key recommendations contains the essential knowledge for most practitioners while the "summary of the summary" at the beginning of this document is worth committing to memory.

This Guideline represents the views of a wide constituency of oxygen users and this in itself has contributed to its complexity. It has followed a robust methodology with careful consideration of the nature of the evidence available although, as the authors make clear, this evidence is largely grade 3 and grade 4 (ie, based on observational clinical studies or expert opinion). This does not mean it is less important but is simply a reflection of the difficulties of conducting appropriate clinical trials in a setting where informed patient consent is often impossible to obtain and where there is a risk that withholding the intervention might seriously disadvantage the patient. It would be impossible and inappropriate to review all the many recommendations in this editorial but some flavour of the scope of what is covered might be helpful.

The Guideline is quite clear that oxygen is given acutely for the treatment of hypoxaemia. This is not the same as giving oxygen to treat breathlessness, as many hypoxaemic patients are not particularly breathless while many breathless patients are not hypoxaemic. Providing patients with a flow of gas over the face may decrease the perception of breathlessness but this mechanism, if it operates in many of the clinical circumstances reviewed here, is certainly not the same as giving oxygen to improve tissue oxygen delivery. Focusing on this more important and better validated use of oxygen has two immediate consequences. The first is that treating tissue hypoxia involves more than just increasing the oxygen concentration. In some circumstances, oxygen delivery is best improved by increasing cardiac output or correcting anaemia and this integrated approach to care based on a proper diagnosis is central to the Guideline recommendations. The second consideration is more practical. Hypoxaemic patients need to be properly identified and it is unwise to rely too much on a clinical diagnosis of central cyanosis. This is less of a problem than in the past as there is a widespread availability of reliable and relatively artefact free pulse oximeters. The section on using oximeters is well worth reading and particularly the pitfalls that follow when measurement is attempted in patients with poor peripheral circulation or even those still wearing nail varnish! Using pulse oximetry and an assessment of the patient's severity, a reasonable inspired oxygen concentration can be selected with the patient's oxygen saturation targeted to maximise benefit and minimise harm.

There is more enthusiasm for diagnosing hypercapnia on clinical grounds, although a good sensitivity and specificity analysis on any of the clinical signs cited is currently lacking. The hazards of hypercapnia in patients with hypoxaemic chronic obstructive pulmonary disease (but also in those with the rarer conditions, such as neuromuscular disease), are appropriately discussed and the proposed target oxygen saturation of $88-92 \%$ in these patients is a sensible recommendation. Even more important is the proposal for a specific oxygen alert card in patients who are at risk of carbon dioxide retention when they receive oxygen or have exhibited this problem in the past. Widespread uptake of this sensible idea would reduce the inadvertent harm done to these patients when transferred to hospital while acutely ill and it is being trialled by many ambulance Trusts in the UK. Although the information contained 